\title{
Extra-abdominal fibromatosis: Clinical and therapeutic considerations based on an illustrative case
}

\author{
ROSARIO FORNARO $^{1}$, ELISA CARATTO ${ }^{1}$, MICHELA CARATTO $^{1}$, ALEXANDER SALERNO $^{1}$, \\ FRANCESCA SAROCCHI $^{2}$, GIUSEPPE MINETTI ${ }^{3}$, MARCO FRASCIO ${ }^{1}$, \\ ROBERTO MURIALDO ${ }^{4}$ and MARIO TAVIANI ${ }^{1}$ \\ Departments of ${ }^{1}$ Surgery, ${ }^{2}$ Anatomical Pathology, ${ }^{3}$ Radiology and ${ }^{4}$ Oncology, IRCCS San Martino Hospital, \\ National Cancer Research Institute, University of Genoa, Genova 16132, Italy
}

Received October 14, 2014; Accepted July 7, 2015

DOI: $10.3892 / 01.2015 .3684$

\begin{abstract}
Extra-abdominal fibromatosis is a rare, benign disease that is characterized by a local but not metastatic invasivity. In particular, desmoid tumors of the chest wall represent only $10-20 \%$ of all deep fibromatoses. The disease occurs more often in females and has a higher incidence between puberty and the fourth decade of life. The present study reports the case of a 34-year-old female who came to our attention due to a voluminous mass in the right subcostal region. The magnetic resonance imaging of the upper abdomen confirmed the presence of a neoplasm localized between the anterior hepatic margin and the right costal plane. Through a right subcostal laparotomy, the voluminous $95 \times 45 \times 94-\mathrm{mm}$ neoplasm was excised. Histological examination showed evidence consistent with extra-abdominal fibromatosis. The patient has not shown recurrence of the disease for 4 years since the surgery. Overall, radical surgery with disease-free resection margins is the prime treatment option for this disease. Other therapeutic options, such as radiotherapy, hormonal therapy or treatment with imatinib mesylate, can also be considered in certain cases.
\end{abstract}

\section{Introduction}

Extra-abdominal fibromatosis, also known as a desmoid tumor, is a rarely observed lesion of benign biological significance, characterized as a non-metastatic lesion with local invasiveness (1). Extra-abdominal fibromatosis represents $<0.03 \%$ of all neoplasms, with an annual incidence of 2-4 cases/1,000,000 individuals, worldwide (1). The most common locations are the shoulder and upper limb (33\%), gluteus and lower extremities (30\%), chest wall and spine

Correspondence to: Professor Rosario Fornaro, Department of Surgery, IRCCS San Martino Hospital, National Cancer Research Institute, University of Genoa, 10 Largo Rosanna Benzi, Genova 16132, Italy

E-mail: rfornaro@unige.it

Key words: extra-abdominal fibromatosis, desmoid tumor, chest wall, histology, surgery
(17\%), and head and neck (10\%) (2). Extra-abdominal fibromatosis occurs more often in females and has a higher incidence between puberty and the fourth decade of life. The tumor originates from the muscle-fascial connective tissue and is therefore also known as deep aponeurotic fibromatosis (1). The desmoid tumor may be primary or secondary to trauma, including surgery, or hormonal stimuli (3). It typically presents as a mass of a hard consistency, with a poorly demarcated margin and poor vascularization, and is commonly infiltrating and adherent to the surrounding tissue. The internal structure of the tumor is composed of abundant collagen material mixed with spindle cells and fibroblasts with abundant eosinophilic cytoplasm (4). Magnetic resonance imaging (MRI) is the gold standard technique for diagnosis (2). The selection of optimal treatment is not standardized but, when possible, radical and aggressive surgical resection with margins free of disease (R0) remains, to date, the preferred treatment for desmoid tumor. However, this treatment still has a fair rate of local recurrence (5).

The present study reports the case of a 34-year-old female with a voluminous mass diagnosed as an extra-abdominal fibromatosis. Written informed consent was obtained from the patient for inclusion in the present study.

\section{Case report}

A 34-year-old female patient was admitted to the Department of Surgery at San Martino Hospital (Genova, Italy) due to a voluminous mass in the right subcostal region that had appeared 4-5 months previously and was associated with gravative pain. The patient's remote medical history reported an endometriosis initially treated with hormonal therapy and then with surgery; in addition, the lesion occurred soon after the birth of the patient's first child. Ultrasonography (US) and thoracoabdominal computed tomography (CT) were performed. US, which was performed due to the difficult accessibility of the anatomical site, identified the presence of a solid mass without central necrosis, that appeared hypoechoic and confounding. Upon CT of the chest, a coarse solid expansive formation was observed. The mass was markedly inhomogeneous with the presence of hyperdense areas indistinguishable from the muscle and bone. MRI of the upper abdomen, with and without 

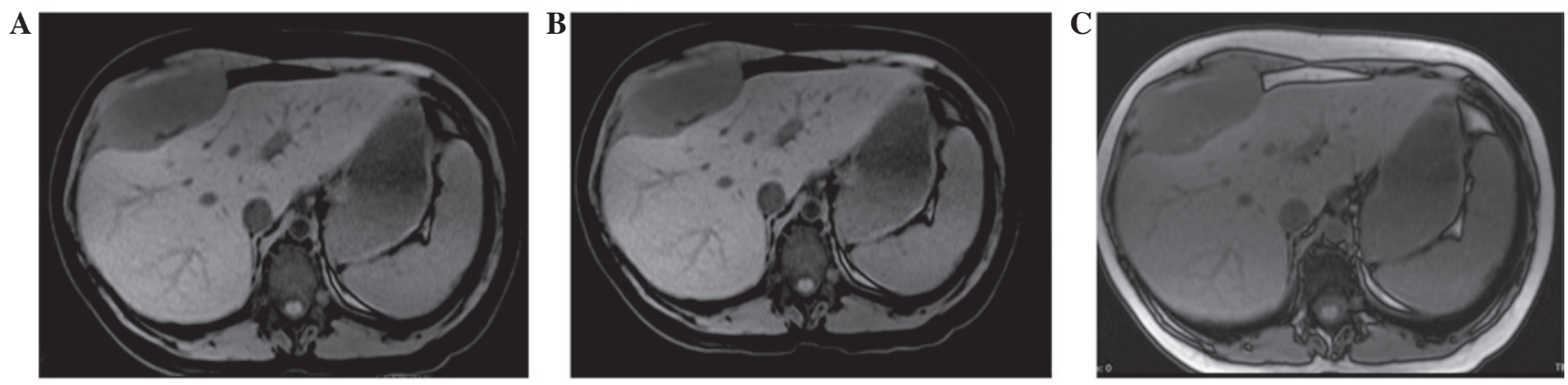

Figure 1. T1-weighted magnetic resonance imaging of the axial plane. (A) T1-weighted imaging in the axial plane showing a 95x45-mm solid mass localized between the liver posteriorly, and the diaphragm and the costal plane anteriorly. No evident loss of signal of the lesion in (B) T1-weighted imaging in opposition phase sequences or (C) fat-saturated T1-weighted imaging, demonstrating the absence of intracellular and extracellular adipose tissue.
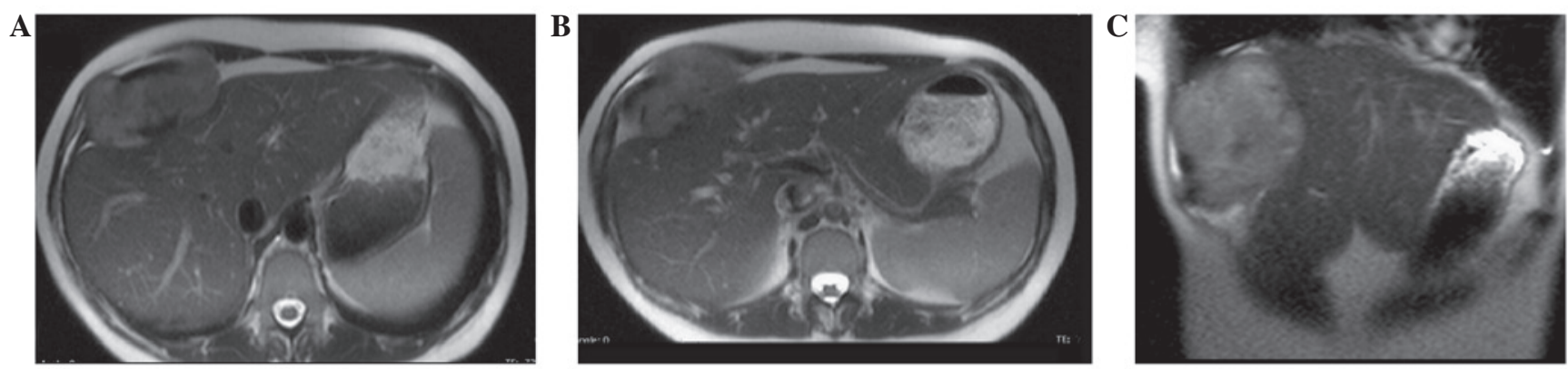

Figure 2. T2-weighted magetic resonance imaging (MRI) showing a non-homogeneous high signal intensity tumor. (A and B) T2-weighted sequences in the axial planes at two different levels confirming the solid nature of the lesion. Herniation of solid tissue through the anterior intercostal spaces is apparent. (C) T2-weighted sequences in the coronal plane confirming the obtuse angles in relation to the liver and aiding in the measurement of the cranio-caudal diameter of the lesion, which appears to be mainly localized in the abdomen.

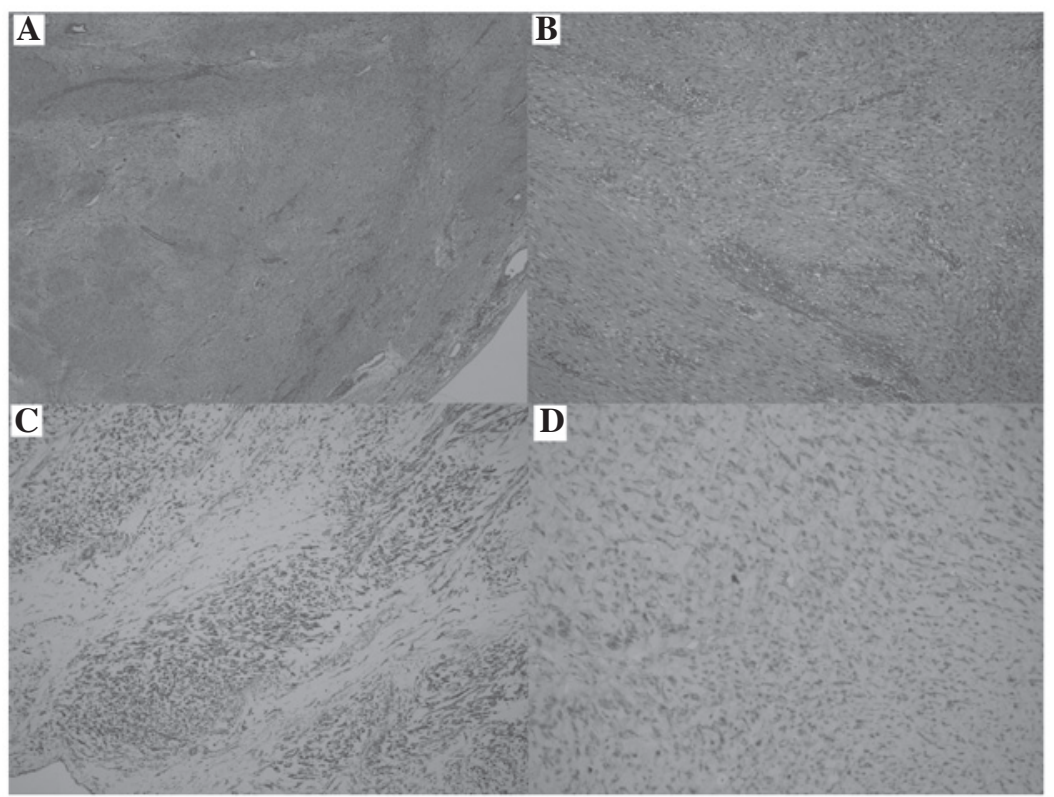

Figure 3. (A) Histological and immunohistochemical examination of the lesion with expansive margins, absence of a true capsule and scattered peripheral chronic inflammatory infiltrations (hematoxylin and eosin staining; magnification, x20). (B) Spindle cell neoplasm disposed in variously orientated bundles, in the absence of mythosis or atypia (hematoxylin and eosin staining; magnification, x200). (C) Immunohistochemical positivity to actin demonstrating the presence of leiomuscolar differentiation of the lesion (magnification, $\mathrm{x} 200$ ). (D) Immunohistochemical nuclear positivity to $\beta$-catenin (magnification, $\mathrm{x} 400$ ).

contrast medium, confirmed the presence of a $95 \times 45 \times 94-\mathrm{mm}$ neoplasm. This was localized between the anterior hepatic margin (dislocated backwards) and the right costal plane, with a strict anatomical association with the diaphragm, to the right rectus abdominis muscle and to the external oblique muscle without a clear cleavage plane. Furthermore, the lesion tended to herniate in the space between the costal cartilages. The lesion appeared to be tenuously hypointense in T1-weighted 
sequences and unevenly hyperintense in T2-weighted sequences, which was not indicative of hematic content, nor pathognomonic of a specific nature (Figs. 1 and 2). The patient therefore underwent a surgical resection. Through a right subcostal laparotomy, the voluminous neoplasm of the right subdiaphragmatic thoracic wall was excised. The mass had developed in the context of the antero-inferior thoracic wall, involving the peritoneum, the diaphragm and the osteomuscular plane of the last four ribs. Once the mass was isolated from the antero-lateral surface of the liver we proceeded with resection of the last four ribs, from the sternum to the posterior axillary line. The thoraco-abdominal wall reconstruction was performed and an intrabdominal-diaphragmatic prosthesis (Dual-Mesh) was positioned to restore the continuity. The intraoperative histological examination evidenced the mesenchymal nature of the lesion and showed that the resection margins were free from disease (R0). The post-operative recovery was regular, and the patient was discharged on day 8 . The definitive histological examination showed evidence consistent with extra-abdominal fibromatosis: The neoplasm was lacking a capsule, with infiltrative-like growth, spindle cells arranged in interlaced bundles and focal mixoid areas, without any significant cytological atypia, a low proliferative index ( $<3 \% \mathrm{Ki}-67$-positive cells) and a very low mitotic index ( $<1$ mitosis/10 high-power fields). No necrotic outbreaks were identified. The immunophenotypic profile showed variable positivity to actin and negativity to desmin, cluster of differentiation (CD)34, CD99, B-cell lymphoma 2, CD117 (c-kit) and S-100 protein (Fig. 3). Although the margins of resection were free from disease (R0), due to the high percentage of recurrences, it was decided to subject the patient to a course of adjuvant radiotherapy (50 Gy in 20 fractions). The use of adjuvant chemotherapy treatment was excluded due to the lack of literature in this regard. The patient, who has been monitored by strict follow-up examinations using CT/MRI at $6,12,24$ and 48 months, has shown no signs of disease recurrence for 4 years since the surgery.

\section{Discussion}

Extra-abdominal fibromatosis, also known as a desmoid tumor, is a rare, non-metastatic and locally invasive lesion that is characterized by a high percentage of local recurrences. The tumor originates from the connective muscle-fascial tissue. Representing $<0.03 \%$ of all neoplasms, the tumor occurs more often in females and can occur in all age ranges, with a peak incidence between puberty and the fourth decade of life (1). Histologically similar desmoid tumors have different prognostic outcomes in relation to age, being locally more aggressive in young subjects, with a higher percentage of recurrences (6). The most common tumor locations are the shoulders and upper limbs (33\%), the gluteus and lower limbs (30\%), the thoracic wall and spinal column (17\%), and the head and neck (10\%) (2). Desmoid tumors can either be primitive or secondary to trauma, including surgical procedures, or hormonal stimulation. Frequently, desmoid tumors occur in young females during or after pregnancy and can regress spontaneously in menopause or after hormonal therapy with tamoxifen (3). In the present case, the patient was suffering from endometriosis and underwent hormonal treatment as well as surgery; in addition to this, the lesion occurred soon after the birth of the patient's first child.

In a fair percentage of cases, fibromatosis is associated with familial adenomatous polyposis (FAP) or Gardner's syndrome $(1,6)$. Despite the multifactorial etiology, certain genetic factors have been identified. For example, in patients affected by FAP or Gardner's syndrome, a mutation in the APC gene has been identified, while in the sporadic forms, there appears to be a mutation in the gene that codes for the $\beta$-catenins. The two mutations cause an increase in the concentration of intracellular $\beta$-catenins, which induces a boost in fibroblastic proliferation (7).

Generally, desmoid tumors present as a mass of hard consistency that is not well-defined, and is typically infiltrative and adherent to the surrounding tissue. The lesion shows poor vascularization and a typical light-brown color, which aids in the differentiation from the adjacent tissue. However, it does not usually exhibit necrotic nor hemorrhagic areas. In the majority of cases, extra-abdominal desmoid tumors are asymptomatic and therefore, at the time of diagnosis, which is mostly incidental, their dimensions usually exceed $10 \mathrm{~cm}$ in diameter (4).

The dimensions of the tumor, its extension and the anatomical association with the surrounding structures can be evaluated with the use of US, CT and MRI, which always must be performed prior to the surgical treatment. Upon US the lesion appears to be hypoechogenic and homogeneous, with variable vascularization. CT shows a soft-tissue mass with different attenuation and enhancement due to the presence of intratumoral hemorrhagic or degenerative areas. The margins often appear to be unclearly defined due to the infiltration of the surrounding tissues $(2,7)$. MRI represents the gold standard to identify the fibromatous lesion and its anatomical association with the adjacent tissues. The technique is also useful in the post-operative follow-up evaluation of the patient (2).

The selection of the optimal treatment is not standardized, above all due to the small number of patients suffering from this disease and due to the few clinical trials that have been conducted. Asymptomatic lesions can be monitored over time, particularly if stable, while treatment is always to be considered in symptomatic patients presenting with lesions either of a large size or that are compressing important vital structures (1). Aggressive surgical resection with safe margins $(2-4 \mathrm{~cm})(\mathrm{R} 0)$ is the prime treatment. An optimal resection of the tumor involving the thoracic wall, as in the present case, is required to include the excision of disease-free ribs, one above and one underneath the lesion (5). It is not always possible to obtain disease-free resection margins, particularly if the tumor involves noble structures such as the spinal column, brachial plexus, major vessels or structures of the fascia of the neck (5). In these cases, a surgical approach is still recommended, even if reductive, to reduce the compressive symptomatology.

In cases where the tumor cannot be operated on and in cases where negative resection margins cannot be obtained, the patient is treated with multimodal therapy with the purpose of reducing the incidence of local recurrences. These treatments include radiotherapy, chemotherapy (anthracycline, vinblastine and methotrexate), hormonal therapy (tamoxifen), non-steroidal anti-inflammatory drugs (NSAIDs), interferon and imatinib mesylate (8). National Comprenhensive Cancer 
Network guidelines suggest the use of post-operative radiotherapy only for tumors of large dimensions and with positive resection margins (9). Maximal doses of radiotherapy are not required to obtain longer periods of disease-free survival (10). There are ongoing studies evaluating the effectiveness of pre-operative radiotherapy in reducing the dimensions of the tumors and in affecting the risk of local recurrence (11). Chemotherapy is used in those patients in whom the tumor exhibits rapid growth or in those who are heavily symptomatic, and it is associated with the use of NSAIDs or COX2 inhibitors. Tamoxifen, an anti-estrogenic agent, is administered in cases where the tumor is inoperable at the same dosages used to treat mammary carcinoma, in virtue of the fact that desmoid tumors are frequently hormone-sensitive. Interferon has been shown to be effective in increasing the period of disease-free survival in certain patients (1). Studies have been conducted on the use of imatinib mesylate (a selective inhibitor of the tyrosine kinase receptor) in the inoperable forms of the tumor and in those patients who refused chemo-radiotherapy or hormonal therapy. At present, study results remain discordant; this is due to the fact that biological factors predictive of response to the drug have not yet been identified (12).

In conclusion, the present study determined that radical resection with margins free of disease still remains the optimal treatment strategy for patients with extra-abdominal fibromatosis. As desmoid tumors are burdened with a high rate of local recurrence, each case should be carefully assessed to evaluate the possibility of using multimodal therapies. In addition, a screening program should be immediately organized to allow careful monitoring of the patient.

\section{References}

1. Escobar C, Munker R, Thoma JO, Li BD and Burton GV: Update on desmoid tumors. Ann Oncol 23: 562-569, 2012.

2. Shinagare AB, Ramaiya NH, Jagannathan JP, Krajewski KM, Giardino AA, Butrynski JE and Raut CP: A to $\mathrm{Z}$ of desmoid tumors. AJR Am J Roentgenol 197: W1008-W1014, 2011.

3. Ioannou M, Demertzis N, Iakovidou I and Kottakis S: The role of imatinib mesylate in adjuvant therapy of extra-abdominal desmoid tumors. Anticancer Res 27: 1143-1147, 2007.

4. Ibrahim M, Sandogji $\mathrm{H}$ and Allam A: Huge intrathoracic desmoid tumor. Ann Thorac Med 4: 146-148, 2009.

5. Mátrai Z, Tóth L, Szentirmay Z, Vámos FR, Klepetko W, Vadász P, Kenessey I and Kásler M: Sporadic desmoid tumors of the chest: Long-term follow-up of 28 multimodally treated patients. Eur J Cardiothorac Surg 40: 1170-1176, 2011.

6. Romero JA, Kim EE, Kim CG, Chung WK and Isiklar I: Different biologic features of desmoid tumors in adult and juvenile patients: MR demonstration. J Comput Assist Tomogr 19: 782-787, 1995.

7. Lamboley JL, Le Moigne F, Proust C, Thivolet-Bejui F, Tronc F, Revel D and Douek P: Desmoid tumour of the chest wall. Diagn Interv Imaging 93: 635-638, 2012.

8. Melis M, Zager JS and Sondak VK: Multimodality management of desmoid tumors: How important is a negative surgical margin? J Surg Oncol 98: 594-602, 2008.

9. National Comprehensive Cancer Network (NCCN). Guidelines for soft tissue sarcomas, Version 1.2011. http://www.ncen. org/professionals/physician_gls/pdf/sarcoma.pdf. Accessed March 13, 2011.

10. Ballo MT, Zagars GK and Pollack A: Radiation therapy in the management of desmoid tumors. Int J Radiat Oncol Biol Phys 42: 1007-1014, 1998.

11. Mankin HJ, Hornicek FJ and Springfield DS: Extra-abdominal desmoid tumors: A report of 234 cases. J Surg Oncol 102: 380-384, 2010.

12. Dufresne A, Bertucci F, Penel N, Le Cesne A, Bui B, Tubiana-Hulin M, Ray-Coquard I, Cupissol D, Chevreau C, Perol D, et al: Identification of biological factors predictive of response to imatinib mesylate in aggressive fibromatosis. $\mathrm{Br} \mathrm{J}$ Cancer 103: 482-485, 2010. 